\title{
Theoretical status of $\varepsilon^{\prime} / \varepsilon$
}

\section{Matthias Jamin* \\ Institut für Theoretische Physik \\ Philosophenweg 16, 69120 Heidelberg, Germany \\ E-mail: 'M. Jamin@ThPhys. Uni-Heidelberg. DE}

ABSTRACT: The present theoretical status of the parameter for direct CP violation $\varepsilon^{\prime} / \varepsilon$ in the Standard Model is reviewed and compared with most recent experimental measurements of the same quantity. After collecting the basic expressions for $\varepsilon^{\prime} / \varepsilon$, the situation of hadronic matrix element calculations is summarised. The matrix elements constitute the dominant source of uncertainty for theoretical determinations of $\varepsilon^{\prime} / \varepsilon$. For central values of the input parameters, the numerical analysis then yields results which are generally below the experimental data. Possible reasons for these findings are discussed.

$\mathrm{T}$ HIS YEAR has experienced a revived strong interest in the parameter $\varepsilon^{\prime} / \varepsilon$ which quantifies direct $\mathrm{CP}$ violation in the neutral kaon system, due to the recent experimental measurements by the KTeV and NA48 collaborations:

$$
\begin{aligned}
& \operatorname{Re}\left(\varepsilon^{\prime} / \varepsilon\right)=(28.0 \pm 4.1) \cdot 10^{-4} \mathrm{KTeV}[1] \\
& \operatorname{Re}\left(\varepsilon^{\prime} / \varepsilon\right)=(18.5 \pm 7.3) \cdot 10^{-4} \mathrm{NA} 48[2]
\end{aligned}
$$

Both measurements confirm the large value found previously by the NA31 collaboration [5] and give rise to the new world average [3]

$$
\operatorname{Re}\left(\varepsilon^{\prime} / \varepsilon\right)=(21.4 \pm 4.0) \times 10^{-4} .
$$

Experimentally, the signature for direct $\mathrm{CP}$ violation is a deviation of the ratio $\left|\eta_{+-} / \eta_{00}\right|^{2}$ from unity, where

$$
\begin{aligned}
\eta_{+-} & \equiv \frac{A\left(K_{L} \rightarrow \pi^{+} \pi^{-}\right)}{A\left(K_{S} \rightarrow \pi^{+} \pi^{-}\right)} \\
\eta_{00} & \equiv \frac{A\left(K_{L} \rightarrow \pi^{0} \pi^{0}\right)}{A\left(K_{S} \rightarrow \pi^{0} \pi^{0}\right)}
\end{aligned}
$$

Theoretically, it is more convenient to consider quantities were the final state pions are in a definite isospin state:

$$
\begin{aligned}
\varepsilon & \equiv \frac{A\left(K_{L} \rightarrow(\pi \pi)_{I=0}\right)}{A\left(K_{S} \rightarrow(\pi \pi)_{I=0}\right)}, \\
\omega & \equiv \frac{A\left(K_{S} \rightarrow(\pi \pi)_{I=2}\right)}{A\left(K_{S} \rightarrow(\pi \pi)_{I=0}\right)} .
\end{aligned}
$$

\footnotetext{
${ }^{*}$ Heisenberg fellow
}

The parameter $\varepsilon$ measures indirect $\mathrm{CP}$ violation in the neutral kaon system which displays the fact that the physical mass eigenstates $K_{L}$ and $K_{S}$ are not eigenstates of $\mathrm{CP}$, but have small admixtures of the order of $\varepsilon$ from the opposite $\mathrm{CP}$ parity. The second parameter $\omega$ has nothing to do with $\mathrm{CP}$ violation, but is introduced for convenience. It parametrises the so called $\Delta I=1 / 2$ rule which states that the isospin zero final state is much enhanced over the isospin two final state. In other words, the $\Delta I=1 / 2$ transition is strongly enhanced over the $\Delta I=3 / 2$ transition.

With the help of these quantities, the parameter $\varepsilon^{\prime}$ can be defined as

$$
\varepsilon^{\prime} \equiv \frac{1}{\sqrt{2}}\left[\frac{A\left(K_{L} \rightarrow(\pi \pi)_{I=2}\right)}{A\left(K_{S} \rightarrow(\pi \pi)_{I=0}\right)}-\varepsilon \cdot \omega\right] .
$$

Using the isospin decomposition of the final twopion state it is a simple exercise to find the relation between the experimental parameters $\eta_{+-}$, $\eta_{00}$ and the theoretical parameters $\varepsilon, \varepsilon^{\prime}$ and $\omega$ :

$$
\begin{gathered}
\eta_{+-}=\varepsilon+\frac{\varepsilon^{\prime}}{1+\omega / \sqrt{2}}, \\
\eta_{00}=\varepsilon-\frac{2 \varepsilon^{\prime}}{1-\sqrt{2} \omega} .
\end{gathered}
$$

From these relations and the measured results for $\eta_{+-}$and $\eta_{00}[4]$ one can deduce an experimental value for $\varepsilon$ :

$$
\varepsilon=(2.280 \pm 0.013) \cdot 10^{-3} e^{i(43.5 \pm 0.1)^{\circ}} .
$$


Employing CPT invariance in addition, the phase has been extracted from the known $K_{L}-K_{S}$ mass difference.

To proceed further, one expresses the parameters $\varepsilon, \varepsilon^{\prime}$ and $\omega$ in terms of isospin amplitudes $A_{I}$ and final state interaction phase shifts $\delta_{I}$ which are defined by

$$
\begin{aligned}
& A\left(K^{0} \rightarrow(\pi \pi)_{I}\right)=i A_{I} e^{i \delta_{I}}, \\
& A\left(\bar{K}^{0} \rightarrow(\pi \pi)_{I}\right)=-i A_{I}^{*} e^{i \delta_{I}} .
\end{aligned}
$$

If CP were conserved, the amplitudes $A_{I}$ would be real. Making use of the relation between the strangeness eigenstates $K^{0}, \bar{K}^{0}$ and the mass eigenstates $K_{L}, K_{S}$ (further details can for example be found in ref. [6]) the parameter $\varepsilon^{\prime}$ can be written as

$$
\varepsilon^{\prime}=\frac{1}{\sqrt{2}}\left(1-\varepsilon^{2}\right) \operatorname{Im}\left(\frac{A_{2}}{A_{0}}\right) e^{i\left(\pi / 2+\delta_{2}-\delta_{0}\right)},
$$

or expressed in a different, equivalent form:

$$
\varepsilon^{\prime}=\frac{\left(1-\varepsilon^{2}\right) e^{i\left(\pi / 2+\delta_{2}-\delta_{0}\right)}}{\sqrt{2}\left(1+\xi_{0}^{2}\right) \operatorname{Re} A_{0}}\left[\operatorname{Im} A_{2}-\xi_{0} \operatorname{Re} A_{2}\right],
$$

where $\xi_{0} \equiv \operatorname{Im} A_{0} / \operatorname{Re} A_{0}$. So far no approximations have been performed and all equations are exact. In particular, equation ( $\left(\overline{1} \overline{3}_{1}^{\prime}\right)$ will be the starting point for our theoretical analysis of the ratio $\varepsilon^{\prime} / \varepsilon$.

Analogously, to a very good approximation the parameter $\omega$ can be expressed as

$$
\omega \approx \frac{\operatorname{Re} A_{2}}{\operatorname{Re} A_{0}} e^{i\left(\delta_{2}-\delta_{0}\right)}=\frac{1}{22.2} e^{-i(45 \pm 6)^{\circ}},
$$

again reflecting the $\Delta I=1 / 2$ rule. The difference of the strong interaction phase shifts has been obtained in ref. [7]. A consequence of the $\Delta I=1 / 2$ rule is that the isospin zero contribution to $\varepsilon^{\prime}$ is suppressed by the small quantity $\omega$ compared to the isospin two component. Phenomenological implications of this observation will be further discussed below.

Using the relations $\left(\overline{\overline{7}_{1}}\right)$ and $\left(\bar{p}_{1} \bar{p}_{1}^{1}\right)$ together with the experimental result (1 $\left.{ }_{1}^{\prime}\right)$ for $\omega$, up to corrections of the order of $2 \%$ one finds

$$
\operatorname{Re}\left(\frac{\varepsilon^{\prime}}{\varepsilon}\right) \approx \frac{1}{6}\left[\left|\frac{\eta_{+-}}{\eta_{00}}\right|^{2}-1\right] .
$$

At present, these corrections are still much below the experimental uncertainties and can be safely neglected.

\section{Basic formulae for $\varepsilon^{\prime} / \varepsilon$}

Neglecting the tiny corrections of order $\varepsilon^{2}$ and $\xi_{0}^{2}$ in equation ( $\left(\overline{3}_{\mathbf{L}}^{\prime}\right)$, the central expression for $\varepsilon^{\prime} / \varepsilon$ takes the form

$$
\frac{\varepsilon^{\prime}}{\varepsilon} \approx \frac{e^{i\left(\pi / 4+\delta_{2}-\delta_{0}\right)}}{\sqrt{2}|\varepsilon| \operatorname{Re} A_{0}}\left[\operatorname{Im} A_{2}-|\omega| \operatorname{Im} A_{0}\right] .
$$

Since it was found that the phase shift difference $\delta_{0}-\delta_{2} \approx \pi / 4$, within the uncertainties $\varepsilon^{\prime} / \varepsilon$ turns out to be real. Calculating the amplitudes $A_{I}$ in the framework of the operator product expansion and applying the renormalisation group equation, the basic formulae for $\varepsilon^{\prime} / \varepsilon$ is found to be

$$
\frac{\varepsilon^{\prime}}{\varepsilon}=\operatorname{Im} \lambda_{t}\left[P^{(1 / 2)}-P^{(3 / 2)}\right] .
$$

Here $\lambda_{t} \equiv V_{t d} V_{t s}^{*}$ with $V_{i j}$ being the elements of the quark mixing or Cabibbo-Kobayashi-Maskawa (CKM) matrix. To an excellent approximation one has

$$
\operatorname{Im} \lambda_{t} \approx\left|V_{u b}\right|\left|V_{c b}\right| \sin \delta
$$

with $\delta$ being the $\mathrm{CP}$-violating phase in the standard parametrisation of the CKM matrix [4].

Further, the $P^{(\Delta I)}$ are given by

$$
\begin{aligned}
P^{(1 / 2)} & =r \sum_{i} y_{i}(\mu)\left\langle(\pi \pi)_{0}\left|Q_{i}(\mu)\right| K\right\rangle\left(1-\Omega_{I B}\right), \\
P^{(3 / 2)} & =\frac{r}{|\omega|} \sum_{i} y_{i}(\mu)\left\langle(\pi \pi)_{2}\left|Q_{i}(\mu)\right| K\right\rangle,
\end{aligned}
$$

with

$$
r=\frac{G_{F}|\omega|}{2|\varepsilon| \operatorname{Re} A_{0}}=346 \mathrm{GeV}^{-3} .
$$

The $y_{i}(\mu)$ are Wilson coefficient functions corresponding to the operators $O_{i}$ and $\mu$ denotes the renormalisation scale which for our analysis will be of order $1 \mathrm{GeV}$. In addition to the renormalisation scale dependence, both, the Wilson coefficients $y_{i}$, and the hadronic matrix elements of operators $O_{i}$, depend on the renormalisation scheme. Of course, up to the calculated order, for the physical quantities $P^{(\Delta I)}$ these dependencies should cancel. Present-day values for the Wilson coefficients $y_{i}\left(m_{c}\right)$ for two commonly used schemes can be found in ref. [8]. Finally, $\Omega_{I B}$ is an isospin breaking correction which arises because $m_{u} \neq m_{d}$. In the numerical analysis we use $\Omega_{I B}=0.25 \pm 0.08[9,10]$ but we shall further comment on isospin breaking effects below. 
Omitting negligible contributions from dimension five magnetic-dipole operators, the leading contribution to $\varepsilon^{\prime} / \varepsilon$ results from dimension six four-quark operators. These can be classified into $Q_{1,2}$ (current-current), $Q_{3-6}$ (QCD penguin) and $Q_{7-10}$ (electroweak penguin) according to the type of Feynman diagrams from which they arise. Explicit expressions for all operators can for example be found in ref. [11] and the review article [12]. Here we only give the two dominant QCD and electroweak penguin operators:

$$
\begin{aligned}
Q_{6} & =\left(\bar{s}_{\alpha} d_{\beta}\right)_{V-A} \sum_{q=u, d, s}\left(\bar{q}_{\beta} q_{\alpha}\right)_{V+A} \\
Q_{8} & =\frac{3}{2}\left(\bar{s}_{\alpha} d_{\beta}\right)_{V-A} \sum_{q=u, d, s} e_{q}\left(\bar{q}_{\beta} q_{\alpha}\right)_{V+A}
\end{aligned}
$$

where $\alpha, \beta$ are colour indices and $e_{q}$ denotes the electric quark charges. Although the contribution of the electroweak penguin $Q_{8}$ is suppressed by a factor $\alpha_{e m}$ it is enhanced by $1 /|\omega|$ and thus, as we shall see further below, has some impact on the value of $\varepsilon^{\prime} / \varepsilon$.

\section{History of $\varepsilon^{\prime} / \varepsilon$ calculations}

To be able to appreciate the achievements of the $\varepsilon^{\prime} / \varepsilon$ calculations in the last almost 25 years since the first estimate of $\varepsilon^{\prime} / \varepsilon$ [13], let me briefly review their history.

The first estimate of $\varepsilon^{\prime} / \varepsilon[13]$ assumed $m_{t} \ll$ $M_{W}$, only included QCD penguins which were introduced first in ref. [14] and omitted renormalisation group effects. Nevertheless, just by chance the resulting value $1 / 450$ is surprisingly close to the current world average. Renormalisation group effects in the leading logarithmic approximation have first been taken into account in [15]. For $m_{t} \ll M_{W}$ only QCD penguins play a substantial role. First extensive phenomenological analyses in this approximation can be found in [16].

Over the eighties these calculations were refined through the inclusion of QED penguin effects for $m_{t} \ll M_{W}[9,17]$, the inclusion of isospin breaking in the quark masses $[9,10]$ and through improved estimates of hadronic matrix elements in the framework of the $1 / N_{c}$ approach [18]. This era of $\varepsilon^{\prime} / \varepsilon$ culminated in the analyses in $[19,20]$, where QCD penguins, electroweak penguins and the relevant box diagrams were included for arbitrary top quark masses. The strong cancellation between QCD penguins and electroweak penguins for $m_{t}>150 \mathrm{GeV}$ found in these papers was later confirmed by other authors [21].

During the nineties considerable progress has been achieved by calculating complete next-toleading order (NLO) corrections to the Wilson coefficients $y_{i}(\mu)[11,22-24]$. Down to a scale of order $1 \mathrm{GeV}$ the corrections turned out to be modest which allows good control over the short distance part in the operator product expansion. Together with the NLO corrections to $\varepsilon$ and $B^{0}$ $\bar{B}^{0}$ mixing [25-27], this allowed for an improved NLO analysis of $\varepsilon^{\prime} / \varepsilon$ including constraints from the observed indirect $\mathrm{CP}$ violation $(\varepsilon)$ and $B_{d, s}^{0}$ $\bar{B}_{d, s}^{0}$ mixings $\left(\Delta M_{d, s}\right)$. Progress in the determination of the $V_{u b}$ and $V_{c b}$ elements of the CKM matrix and in particular the determination of the top quark mass $m_{t}$ had of course also an important impact on $\varepsilon^{\prime} / \varepsilon$.

Nevertheless, it is fair to say that calculations of the long distance part, the hadronic matrix elements, have not yet reached a level which would match the NLO calculations of the Wilson coefficients. Long distance physics inevitably involves confinement effects and thus non-perturbative methods are required for the calculation of the matrix elements. In principal lattice QCD calculations should be able to give matrix elements with the correct scale and scheme dependencies to match the coefficient functions, but the most important matrix element of $Q_{6}$ has so far not been obtained reliably.

Other methods which are based on effective theories like chiral perturbation theory (ChPT) or the $1 / N_{c}$ expansion suffer from problems because a sound matching to the Wilson coefficients with the correct scale and especially scheme dependencies is not obvious. Finally, QCD sum rules could be useful, but except for the $K^{0}-\bar{K}^{0}$ mixing parameter $B_{K}$, the calculation of hadronic matrix elements in this approach has not been developed far enough to be competitive to the other methods. In the next section we shall thus summarise the status of the hadronic matrix elements relevant for $\varepsilon^{\prime} / \varepsilon$ concentrating on the dominant contributions $\left\langle Q_{6}\right\rangle_{0}$ and $\left\langle Q_{8}\right\rangle_{2}$. 


\section{Hadronic matrix elements}

For a discussion of the hadronic matrix elements it is convenient to introduce the so called $B$-parameters which quantify the deviation of the full matrix elements to the vacuum-saturation or factorisation approximation were the four-quark operator is factorised in the product of two currents by inserting a vacuum intermediate state. This approximation usually serves as a first estimate of hadronic matrix elements. Thus we define

$$
\begin{aligned}
& \left\langle Q_{6}\right\rangle_{0} \equiv B_{6}^{(1 / 2)}\left\langle Q_{6}\right\rangle_{0}^{(v a c)}, \\
& \left\langle Q_{8}\right\rangle_{2} \equiv B_{8}^{(3 / 2)}\left\langle Q_{8}\right\rangle_{2}^{(v a c)},
\end{aligned}
$$

and the factorisation approximation corresponds to $B_{6}^{(1 / 2)}=B_{8}^{(3 / 2)}=1$. These values even hold in the large- $N_{c}$ limit [18] which means that in this limit factorisation is exact.

Because it turns out that the factorised matrix elements $\left\langle Q_{6}\right\rangle_{0}^{(v a c)}$ and $\left\langle Q_{8}\right\rangle_{2}^{(v a c)}$ are proportional to $1 / m_{s}^{2}$, the value of the strange quark mass enters the analysis of $\varepsilon^{\prime} / \varepsilon$. This is not necessary as for example on the lattice the matrix elements $\left\langle Q_{6}\right\rangle_{0}$ and $\left\langle Q_{8}\right\rangle_{2}$ are calculated directly and no direct dependence on the strange mass arises. However, although the matrix elements depend on the renormalisation scale, a careful analysis [11] showed that this dependence is almost completely covered by the scale dependence of the strange mass and $B_{6}^{(1 / 2)}, B_{8}^{(3 / 2)}$ for energies of interest are practically scale independent. In fact, this statement is exact in the large- $N_{c}$ limit since in this limit the anomalous dimensions of $Q_{6}$ and $Q_{8}$ are minus twice the mass anomalous dimension [11]. For this reason we stick to the discussion of the $B$-parameters which make a comparison of different methods which work at different scales easier.

The status of strange quark mass determinations has been recently summarised in refs. $[8,28,29]$. For further references the reader is referred to these works. Most precise values of the strange quark mass come from lattice QCD and QCD sum rule calculations. As a present average, we quote

$$
m_{s}(2 \mathrm{GeV})=(110 \pm 20) \mathrm{MeV} .
$$

Unquenched lattice calculations yield somewhat smaller values but at present the information is

\begin{tabular}{|c|c|c|}
\hline Method & $B_{6}^{(1 / 2)}$ & $B_{8}^{(3 / 2)}$ \\
\hline Lattice $[30]$ & - & $0.69-1.06$ \\
Large- $N_{c}[31]$ & $0.72-1.10$ & $0.42-0.64$ \\
ChQM [32] & $1.07-1.58$ & $0.75-0.79$ \\
\hline
\end{tabular}

Table 1: Results for $B_{6}^{(1 / 2)}$ and $B_{8}^{(3 / 2)}$ obtained in different approaches.

not precise enough to be conclusive. In the approach to the analysis of $\varepsilon^{\prime} / \varepsilon$ of ref. [11] it is convenient to calculate the matrix elements at the scale $m_{c}$ because at that scale many of the remaining hadronic matrix elements can be determined from CP-conserving $K \rightarrow \pi \pi$ decays. Thus we also present the value of the strange mass at that scale:

$$
m_{s}\left(m_{c}\right)=(130 \pm 25) \mathrm{MeV},
$$

where $m_{c}=1.3 \mathrm{GeV}$ has been used.

Values for $B_{6}^{(1 / 2)}$ and $B_{8}^{(3 / 2)}$ obtained in various approaches are collected in table 1, 1, The lattice results have been calculated at $\mu=2 \mathrm{GeV}$. Concerning the lattice results for $B_{6}^{(1 / 2)}$, old calculations gave values around one with errors of the order of $30 \%$. However, a recent work [33] shows that NLO QCD corrections in the relation between lattice and continuum operators are so huge that at present there is no solid prediction for $B_{6}^{(1 / 2)}$ on the lattice. The situation of $B_{8}^{(3 / 2)}$ is better. Here most approaches find a suppression of $B_{8}^{(3 / 2)}$ below unity by roughly $20 \%$. Further discussion of the lattice approach and additional references can be found in [34].

The average value of $B_{6}^{(1 / 2)}$ in the large- $N_{c}$ approach including full order $p^{2}$ and $p^{0} / N_{c}$ contributions as given in table $\overline{1}_{1}^{1}$ is close to 1 whereas the suppression of $B_{8}^{(3 / 2)}$ compared to the large$N_{c}$ limit is stronger than on the lattice. The uncertainty comes from a variation of the cut-off scale $\Lambda$ in the effective theory. On the other hand it has been found [35] that a higher order term $\mathcal{O}\left(p^{2} / N_{c}\right)$ enhances $B_{6}^{(1 / 2)}$ to 1.6. This result is clearly interesting. Yet, in view of the fact that other $p^{2} / N_{c}$ terms as well as $p^{4}$ and $p^{0} / N_{c}^{2}$ terms have not been calculated, it is premature to take this enhancement seriously.

Finally, the chiral quark model (ChQM) gives values for $B_{6}^{(1 / 2)}$ as high as $1.33 \pm 0.25$. On the 
other hand, $B_{8}^{(3 / 2)}$ in this approach is well compatible with the lattice and large- $N_{c}$ calculations. Guided by the results presented above and biased to some extent by the results from the lattice and large- $N_{c}$ approach to hadronic matrix elements, the status of determinations of $B_{6}^{(1 / 2)}$ and $B_{8}^{(3 / 2)}$ is summarised as:

$$
B_{6}^{(1 / 2)}=1.0 \pm 0.3, \quad B_{8}^{(3 / 2)}=0.8 \pm 0.2 .
$$

In addition, in our numerical analysis we shall always keep $B_{6}^{(1 / 2)} \geq B_{8}^{(3 / 2)}$ as it is found with all non-perturbative methods.

\section{Numerical analysis of $\varepsilon^{\prime} / \varepsilon$}

Being one of the authors of a recent analysis of $\varepsilon^{\prime} / \varepsilon[8]$ by the so-called Munich group, I take the liberty to mainly concentrate on this work but I shall comment on other recent analyses below.

Before we can proceed with the calculation of $\varepsilon^{\prime} / \varepsilon$, we still have to extract the value of $\sin \delta$, or equivalently, $\operatorname{Im} \lambda_{t}$ directly. This can be obtained from a standard analysis of the unitarity triangle which uses data for $\left|V_{c b}\right|,\left|V_{u b}\right|, \varepsilon, \Delta M_{d}$ and $\Delta M_{s}$, where the last two measure the size of $B_{d, s}^{0}-\bar{B}_{d, s}^{0}$ mixing. This type of analysis is rather well known and the reader is referred to the literature for details $[34,36,37]$. As our result for $\operatorname{Im} \lambda_{t}$, we quote [8]

$$
\operatorname{Im} \lambda_{t}=(1.33 \pm 0.14) \cdot 10^{-4} .
$$

At this point it is instructive to present a formula which is not to be used for any serious analysis, nevertheless in a crude approximation displays the dependence of $\varepsilon^{\prime} / \varepsilon$ on the most important parameters collected in table $\overline{2}_{2}$ :

$$
\begin{gathered}
\frac{\varepsilon^{\prime}}{\varepsilon} \approx 13 \operatorname{Im} \lambda_{t}\left[\frac{130 \mathrm{MeV}}{m_{s}\left(m_{c}\right)}\right]^{2}\left[B_{6}^{(1 / 2)}\left(1-\Omega_{I B}\right)\right. \\
\left.-0.4 B_{8}^{(3 / 2)}\left(\frac{m_{t}\left(m_{t}\right)}{165 \mathrm{GeV}}\right)^{2.5}\right]\left(\frac{\Lambda_{\overline{\mathrm{MS}}}^{(4)}}{340 \mathrm{MeV}}\right) .(4.2)
\end{gathered}
$$

This formula exhibits clearly the dominant uncertainties which reside in the values of $B_{6}^{(1 / 2)}$, $B_{8}^{(3 / 2)}, m_{s}, \Lambda \frac{(4)}{\mathrm{MS}}$ and $\Omega_{I B}$. Because of the rather accurate value of the top quark mass, the resulting uncertainty in $\varepsilon^{\prime} / \varepsilon$ amounts only to a few percent.

\begin{tabular}{|c|c|c|}
\hline Quantity & Value & Reference \\
\hline$\Lambda \frac{(4)}{\mathrm{MS}}$ & $(340 \pm 50) \mathrm{MeV}$ & {$[4]$} \\
$m_{s}\left(m_{c}\right)$ & $(130 \pm 25) \mathrm{MeV}$ & See text \\
$m_{t}\left(m_{t}\right)$ & $(165 \pm 5) \mathrm{GeV}$ & {$[4]$} \\
$B_{6}^{(1 / 2)}$ & $1.0 \pm 0.3$ & See text \\
$B_{8}^{(3 / 2)}$ & $0.8 \pm 0.2$ & See text \\
\hline
\end{tabular}

Table 2: Collection of main input parameters.

Let us now continue with the full analysis. Using equations (11.2i)-(1) ues for the Wilson coefficient functions $y_{i}[8,11]$, the values of the $B$-parameters and strange quark mass as discussed in the previous section, expressions for the matrix elements in the vacuum insertion approximation $[8,11]$, as well as the value of $\Omega_{I B}$ given in section 2 , we are in a position to calculate $\varepsilon^{\prime} / \varepsilon$.

For an estimation of the uncertainties in the determination of $\varepsilon^{\prime} / \varepsilon$ we follow two different strategies:

- Method 1: All experimental and theoretical input parameters are scanned independently within their ranges to produce the minimal and maximal value for $\varepsilon^{\prime} / \varepsilon$.

- Method 2: A Monte Carlo analysis is performed were all experimental input parameters are simulated with Gaussian errors and all theoretical input parameters with flat errors. The result for $\varepsilon^{\prime} / \varepsilon$ is then extracted from a statistical analysis of the resulting probability distribution.

In the so-called NDR scheme, our result for the scanning method is:

$$
1.05 \cdot 10^{-4} \leq \varepsilon^{\prime} / \varepsilon \leq 28.8 \cdot 10^{-4} .
$$

The values found in the HV scheme, a second scheme considered by us, are generally $20-30 \%$ lower. This reflects the fact that at present the scheme dependence of the matrix elements is not fully under control and the difference in the results is due to a residual scheme dependence. For the statistical analysis in the NDR scheme, we obtain

$$
\varepsilon^{\prime} / \varepsilon=\left(7.7_{-3.5}^{+6.0}\right) \cdot 10^{-4},
$$

were similar comments apply for the result in the HV scheme. We have quoted the median and 


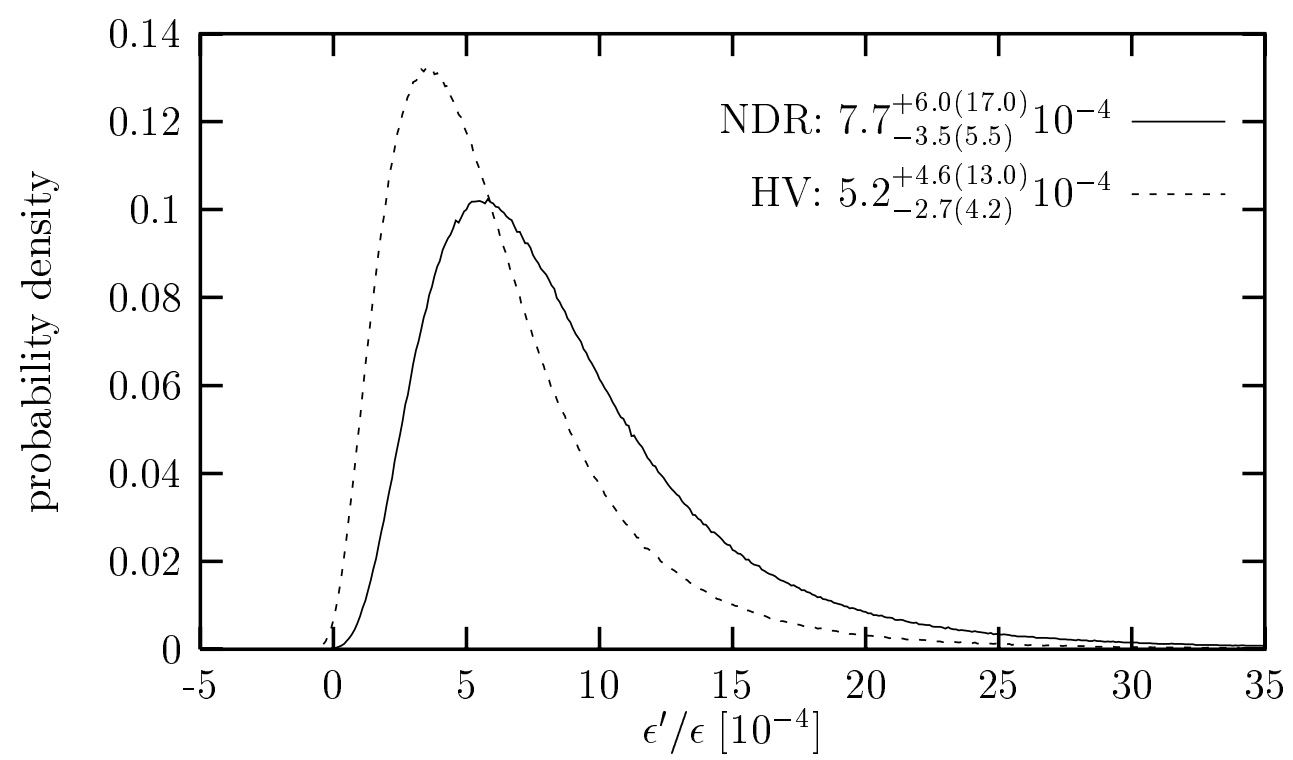

Figure 1: Probability distribution for $\varepsilon^{\prime} / \varepsilon$ in the NDR and HV schemes.

$68 \%$ confidence intervals because the resulting distribution is rather asymmetric. A plot of the corresponding probability distribution is shown in figure $\overline{1}_{1}^{1}$

In table ${ }^{-1}$ we show the values of $\varepsilon^{\prime} / \varepsilon$ in units of $10^{-4}$ for specific values of $B_{6}^{(1 / 2)}, B_{8}^{(3 / 2)}$ and $m_{s}\left(m_{c}\right)$ as calculated in the NDR scheme. The corresponding values in the $\mathrm{HV}$ scheme are lower as discussed above. The fourth column shows the results for central values of all remaining parameters. The comparison of the fourth and fifth column demonstrates how $\varepsilon^{\prime} / \varepsilon$ is increased when $\Lambda \frac{(4)}{\mathrm{MS}}$ is raised from $340 \mathrm{MeV}$ to $390 \mathrm{MeV}$. As stated in equation (4.2), $\varepsilon^{\prime} / \varepsilon$ is roughly proportional to $\Lambda_{\overline{\mathrm{MS}}}^{(4)}$. Finally, in the last column maximal values of $\varepsilon^{\prime} / \varepsilon$ are given. To this end we have scanned all parameters relevant for the analysis of $\operatorname{Im} \lambda_{t}$ within one standard deviation and have chosen $\Lambda_{\overline{\mathrm{MS}}}^{(4)}=390 \mathrm{MeV}$. Comparison of the last two columns demonstrates the impact of the increase of $\operatorname{Im} \lambda_{t}$ from its central to its maximal value and the variation of $m_{t}$.

We observe that the most probable values for $\varepsilon^{\prime} / \varepsilon$ are in the ball park of $10^{-3}$. On the other hand table $\overline{3}$ shows that for particular choices of input parameters, values for $\varepsilon^{\prime} / \varepsilon$ as high as $(2-$ $3) \cdot 10^{-3}$ cannot be excluded. The largest uncertainties reside in $m_{s}, B_{6}^{(1 / 2)}$ and $B_{8}^{(3 / 2)} . \varepsilon^{\prime} / \varepsilon$ increases by roughly a factor of 2.3 when $m_{s}\left(m_{c}\right)$ is changed from $155 \mathrm{MeV}$ to $105 \mathrm{MeV}$. The increase of $B_{6}^{(1 / 2)}$ from 1.0 to 1.3 increases $\varepsilon^{\prime} / \varepsilon$ by approximately $60 \%$ whereas corresponding changes due to $B_{8}^{(3 / 2)}$ are around $40 \%$. The combined uncertainty due to $\operatorname{Im} \lambda_{t}$ and $m_{t}$ are roughly $25 \%$ and the uncertainty coming from $\Lambda_{\overline{\mathrm{MS}}}^{(4)}$ amounts to $15 \%$.

In figure $\overline{2}_{-1}^{1}$ we show the minimal value of $B_{6}^{(1 / 2)}$ for two choices of $m_{s}\left(m_{c}\right)$ and $\Lambda_{\overline{\mathrm{MS}}}^{(4)}$ as a function of $B_{8}^{(3 / 2)}$ for which the theoretical value of $\varepsilon^{\prime} / \varepsilon$ is higher than $2 \cdot 10^{-3}$. To obtain this plot we have varied all other parameters within their uncertainties. We show also a line which corresponds to the relation

$$
B_{6}^{(1 / 2)}=1.7 \cdot B_{8}^{(3 / 2)} .
$$

This relation holds in the large- $N_{c}$ approach to the hadronic matrix elements independent of the cut-off scale $\Lambda$. One observes that as long as $B_{8}^{(3 / 2)} \geq 0.6$, the parameter $B_{6}^{(1 / 2)}$ is required to be larger than unity.

Let me now come to a comparison with other recent analyses of $\varepsilon^{\prime} / \varepsilon$. All groups use the Wilson coefficient functions calculated in refs. [11,22-24]. Therefore, the differences in $\varepsilon^{\prime} / \varepsilon$ result dominantly from different values for the hadronic matrix elements and to some extent different input parameters needed for the determination of $\operatorname{Im} \lambda_{t}$. 


\begin{tabular}{|c|c|c|c|c|c|}
\hline$B_{6}^{(1 / 2)}$ & $B_{8}^{(3 / 2)}$ & $m_{s}\left(m_{c}\right)[\mathrm{MeV}]$ & Central & $\Lambda_{\frac{\mathrm{MS}}{(4)}}=390 \mathrm{MeV}$ & Maximal \\
\hline \multirow{3}{*}{1.3} & \multirow{3}{*}{0.6} & 105 & 20.2 & 23.3 & 28.8 \\
\hline & & 130 & 12.8 & 14.8 & 18.3 \\
\hline & & 155 & 8.5 & 9.9 & 12.3 \\
\hline \multirow{3}{*}{1.3} & \multirow{3}{*}{0.8} & 105 & 18.1 & 20.8 & 26.0 \\
\hline & & 130 & 11.3 & 13.1 & 16.4 \\
\hline & & 155 & 7.5 & 8.7 & 10.9 \\
\hline \multirow{3}{*}{1.3} & \multirow{3}{*}{1.0} & 105 & 15.9 & 18.3 & 23.2 \\
\hline & & 130 & 9.9 & 11.5 & 14.5 \\
\hline & & 155 & 6.5 & 7.6 & 9.6 \\
\hline \multirow{3}{*}{1.0} & \multirow{3}{*}{0.6} & 105 & 13.7 & 15.8 & 19.7 \\
\hline & & 130 & 8.4 & 9.8 & 12.2 \\
\hline & & 155 & 5.4 & 6.4 & 7.9 \\
\hline \multirow{3}{*}{1.0} & \multirow{3}{*}{0.8} & 105 & 11.5 & 13.3 & 16.9 \\
\hline & & 130 & 7.0 & 8.1 & 10.4 \\
\hline & & 155 & 4.4 & 5.2 & 6.6 \\
\hline \multirow{3}{*}{1.0} & \multirow{3}{*}{1.0} & 105 & 9.4 & 10.9 & 14.1 \\
\hline & & 130 & 5.5 & 6.5 & 8.5 \\
\hline & & 155 & 3.3 & 4.0 & 5.2 \\
\hline
\end{tabular}

Table 3: Values of $\varepsilon^{\prime} / \varepsilon$ in units of $10^{-4}$ for specific values of $B_{6}^{(1 / 2)}, B_{8}^{(3 / 2)}, m_{s}\left(m_{c}\right)$ and other parameters as explained in the text.

A very recent analysis by the Rome group [34], besides $B_{6}^{(1 / 2)}$ using matrix elements from the lattice in the HV scheme, finds $\varepsilon^{\prime} / \varepsilon=(4-$ $7) \cdot 10^{-4}$, completely compatible with the results presented above in the statistical analysis. Also their scanning results are similar except that the Rome group does not use the constraint $B_{6}^{(1 / 2)} \geq$ $B_{8}^{(3 / 2)}$ and allows for a larger error in $B_{6}^{(1 / 2)}$ which results in values as low as $\varepsilon^{\prime} / \varepsilon=-1 \cdot 10^{-3}$.

Matrix elements in the large- $N_{c}$ approach were used in the analysis of $\varepsilon^{\prime} / \varepsilon$ by the Dortmund group [35]. With the exception of the large correction of $\mathcal{O}\left(p^{2} / N_{c}\right)$ which was found to enhance $B_{6}^{(1 / 2)}$ up to 1.6 , the matrix elements in the large- $N_{c}$ approach are in agreement to the values used in our analysis. Thus, of course, also the resulting values for $\varepsilon^{\prime} / \varepsilon$ agree and the larger value for $B_{6}^{(1 / 2)}$ would bring $\varepsilon^{\prime} / \varepsilon$ much closer to the experimental average.

Finally, the Trieste group [32] generally finds higher values of $\varepsilon^{\prime} / \varepsilon$, with the central value around $17 \cdot 10^{-4}$ and consequently consistent with the experimental findings. The main reason is a higher value of $B_{6}^{(1 / 2)}$ as obtained from the chiral quark model. In principal one could compare the re- sults in the large- $N_{c}$ and ChQM approaches, but, whereas the former was regularised with a cutoff, in the latter calculation dimensional regularisation was used and a direct comparison is not possible.

\section{Discussion}

As the numerical analysis above shows, for present values of the theoretical input parameters, estimates of $\varepsilon^{\prime} / \varepsilon$ in the Standard Model are typically below the experimental data. However, as the scanning analysis demonstrates, for suitably chosen parameters $\varepsilon^{\prime} / \varepsilon$ in the Standard Model can be made consistent with the data. Yet, this only happens if several of the relevant parameters are simultaneously close to extreme values of the ranges given in table 2 . On the other hand also $B_{6}^{(1 / 2)} \approx 2$ would bring $\varepsilon^{\prime} / \varepsilon$ in agreement with the measured value for central values of the other parameters. Let us further discuss possible scenarios within the Standard Model which would yield consistency of $\varepsilon^{\prime} / \varepsilon$ with the experimental measurements without requiring additional new physics contributions. 


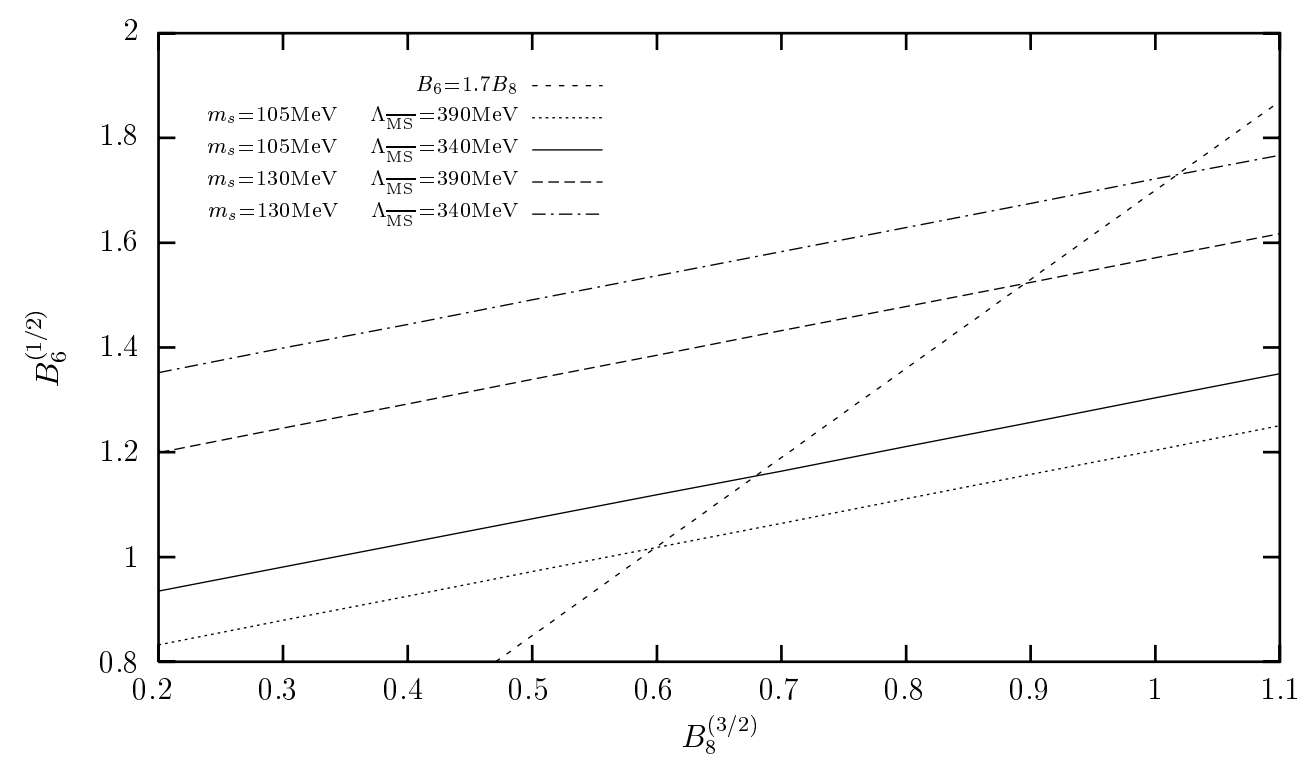

Figure 2: Minimal value for $B_{6}^{(1 / 2)}$ consistent with $\varepsilon^{\prime} / \varepsilon \geq 2 \cdot 10^{-3}$ as a function of $B_{8}^{(3 / 2)}$.

The calculations of the $B$-parameters $B_{6}^{(1 / 2)}$ and $B_{8}^{(3 / 2)}$ involve non-perturbative physics and are thus still very uncertain. Whereas the value of $B_{8}^{(3 / 2)}$ seems to be under better theoretical control, it could well be that the ranges as given in table by the large correction of $\mathcal{O}\left(p^{2} / N_{c}\right)$ in the large$N_{c}$ approach. Additional indications in this direction come from the recent work in refs. [38]. Also using large- $N_{c}$ methods and an intermediate colour-singlet boson which is claimed to provide the correct matching between the shortdistance Wilson coefficients and the hadronic matrix elements, the authors of [38] obtain $B_{6}^{(1 / 2)}=$ $2.2 \pm 0.5$. Although premature at this stage the result is certainly interesting as it would provide the required enhancement of $\varepsilon^{\prime} / \varepsilon$.

Another contribution to $\varepsilon^{\prime} / \varepsilon$ which deserves to be reconsidered are the isospin-breaking corrections. The original calculations $[9,10]$ which more than ten years ago estimated $\Omega_{I B} \approx 0.25$, only considered $\pi^{0}-\eta, \eta^{\prime}$ mixing as the source for isospin-breaking. As pointed out in the recent work [39], additional isospin-violating effects arise from the $u$ - $d$ quark mass difference directly. Estimating these additional contributions in chiral perturbation theory with resonances [40] the authors of [39] find that $\Omega_{I B}$ might even change sign and become as low as $\Omega_{I B} \approx-0.6$, depending on the couplings of the scalar resonance sector. Such a change effectively would correspond to $B_{6}^{(1 / 2)} \approx 2$, again bringing $\varepsilon^{\prime} / \varepsilon$ in agreement with the experimental average. However, in this case the couplings of the scalar resonances are rather uncertain and the findings in ref. [39] need further corroboration.

The final point that should be discussed here is the issue of final state interactions. In principle, non-perturbative approaches to the hadronic matrix elements should also reproduce the strong final-state phases of the $\pi \pi$ system. At present, however, since these phases are generated by chiral loops, in all approaches to the matrix elements they are either zero [18], or found substantially smaller than the experimental values $[31,32]$. A first step in the direction to fully include final-state interaction effects in the calculation of $\varepsilon^{\prime} / \varepsilon$ has been taken very recently in ref. [41].

In the elastic region for the $\pi \pi$ scattering, unitarity and analyticity constraints permit to give a representation of the isospin amplitudes $A_{I}$ in terms of the so-called Omnès integral [42] which involves the phase shifts $\delta_{I}$, times an arbi- 
trary polynomial in momenta. Thus, the effects of chiral logarithms are resummed to all orders in the Omnès integral and the polynomial ambiguity can in principal be fixed by a calculation in chiral perturbation theory. Taking these steps with lowest order chiral expressions for the polynomial ambiguity, the authors of [41] found that besides the imaginary parts which reproduce the final state phases by construction, there is also a substantial enhancement of the real part for isospin zero and a slight suppression for isospin two. The corresponding enhancement and suppression factors $\Re_{I}$ were estimated to be

$$
\Re_{0}=1.41 \pm 0.06, \quad \Re_{2}=0.92 \pm 0.02,
$$

respectively. Applying these factors in the expression for $\varepsilon^{\prime} / \varepsilon$, for central values of the parameters $\varepsilon^{\prime} / \varepsilon=15 \cdot 10^{-4}$, much closer to the experimental result.

As they stand, the results by the authors of ref. [41] are very interesting and may provide the dominant source of enhancement required to bring theoretical calculations of $\varepsilon^{\prime} / \varepsilon$ within the Standard Model and the experimental results into

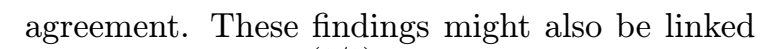

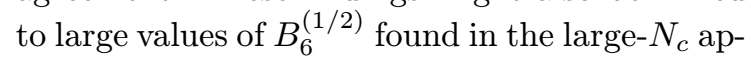
proach. Nevertheless, it would be important to demonstrate that the factors $\Re_{I}$ take the values of equation (5.1.) also in the calculation of individual matrix elements in particular approaches like large- $N_{c}$ or the chiral quark model.

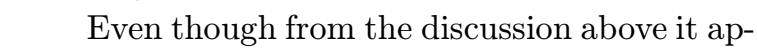
pears that new-physics contributions at present are not required to fit the data for $\varepsilon^{\prime} / \bar{\varepsilon}$, there is certainly still room for such contributions. The most plausible sizable contribution could come from chromo-magnetic penguins in general su- persymmetric models or modiffed- $Z$-penguins.- $O n-$ '- the- other hand substantial mođodifications"of $\mathrm{QCD}^{-}$ penguins through new physics are rather improbable. For a further discussion of contributions to $\varepsilon^{\prime} / \varepsilon$ beyond the Standard Model, the reader is - referered to the talk by _Masiero_ [43].

The future of $\varepsilon^{\prime} / \varepsilon$ in the Standard Model

'- ard -its- extensiens wrill -depend -strongly on-theprogress which is reached in the calculation of hadronic matrix elements. This progress should include a reliable calculation of $B_{6}^{(1 / 2)}$ on the lattice, control over the scale and scheme dependen- cies in approaches using effective low energy theories such that a proper matching with the Wilson coefficient functions at the next-to-leading order will be possible, a better understanding of isospin-breaking effects, and finally, the proper inclusion of final state interactions. First successes in all these areas have been achieved and, together with the upcoming improvements of the experimental measurements with increased data sets, to my mind the future for $\varepsilon^{\prime} / \varepsilon$ in the new millennium looks bright.

\section{Acknowledgments}

It is a pleasure to thank my collaborators for a most enjoyable time working on $\varepsilon^{\prime} / \varepsilon$ and the organisers for a very pleasant and interesting meeting on heavy flavour physics. I would also like to thank the Deutsche Forschungsgemeinschaft for their support.

\section{References}

[1]_A. Alavi-Harati et al. (KTeV), 'Phys. Rev. Lett.' $83-(1999) 2 \overline{2}$

[2] V. Fanti et al. (NA48), hep-ex/9909022.

[3] G. D'Agostini, hepe-ex/9910036,

[4] Review of Particle Properties, Eur. Phys. J. C 3 (1998) 1.

[5] H. Burkhardt et al. (NA31), Phhys. Lett. B-206' (1988) 169 , G.D. Barr et al. (NA31), Phyys. Lett: B 317_(1993) 233i.

[6] E. de Rafael, Lectures given at the 1994 TASISchool on CP violation and limits of the Standard Model, (Boulder, Colorado, June 1994).

[7]_ J. Gasser and U.-G. Meissner, 'Phys. Lett. B $25 \overline{8}$, (1991) 219

[8] S. Bosch, A.J. Buras, M. Gorbahn, S. Jäger, M. Jamin, M.E. Lautenbacher and L. Silvestrini, hep-ph/9904408, to appear in Nucl. Phys. B.

[9] J.F. Donoghue et al., iPhys. Lett. B $\mathbf{1} \overline{\mathbf{7}} \mathbf{9}(\mathbf{1 9 8 6 )}$ $361_{r}^{\prime}$ A.J. Buras and J.M. Gérard, 'Phys. Lett.:

B $192(1987) 156$.

[10] H.-Y. Cheng, Physs.Lett. B 201 (1988) 155; M. Lusignoli, №cl. Phys. B 325 (1989) 33i

[11] A.J. Buras, M. Jamin and M.E. Lautenbacher, Nucl. Phys. B $408(1993) 209$ 
[12] G. Buchalla, A.J. Buras and M.E. Lautenbacher, Rev. Mod. Phys. 68 (1996)_1125.

, [13] - J. -Ellis,-MI-K.- Gaillard- and D. D.

:- -

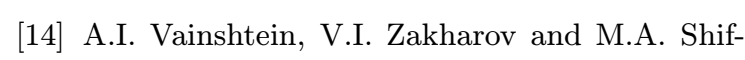
man, Sov. Phys. JETP Lett. 22 (1975)_55'; 'Sov.' F-Dhys.-LETP Lett.-45 (1977)-67-0

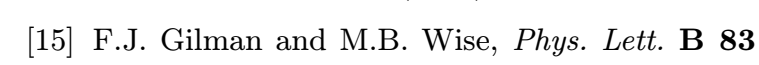
(1979) 83; B. Guberina and R.D. Peccei, №cl. :-2-2hy.-B-168-(1980)-289.

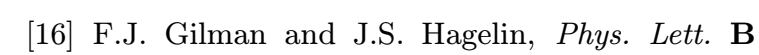

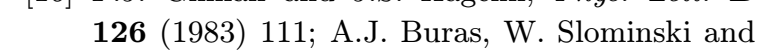

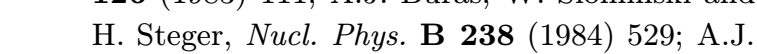
Buras and J.-M. Gérard, Phys. Lett. B 203 - -

[17] J. Bijnens and M.B. Wise, 'P $\bar{L} \bar{y}$ s. Lett. B $\overline{\mathbf{1}} \overline{\mathbf{3}} \overline{\mathrm{7}}$, (1984) 245

[18] W.A. Bardeen, A.J. Buras and J.-M. Gérard, iPhys. Lett. B 180 (1986) 133'; 'Nucl. Phys. B' - - _ 293 (1987) 7877; 'Phys. Lett. B 192 (1987)_138'.

[19] J.M. Flynn and L. Randall, 'Phys. Lett. B - 224, (1989) 2215 erratum iPhys. Lett. B 235 (1990)! - - - 412 .

[20] G. Buchalla, A.J. Buras and M.K. Harlander, 'Nucl. Phys. B $\mathbf{3} \overline{3} 7$ (1990) 313i.

[21] E.A. Paschos and Y.L. Wu, 'Mod. Phys. Lett. A' ' 6 (1991) 93; M. Lusignoli, L. Maiani, G. Mar-

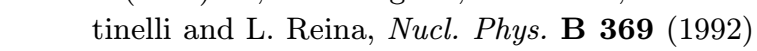

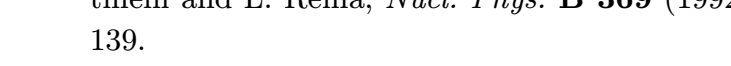

[22] A.J. Buras, M. Jamin, M.E. Lautenbacher and

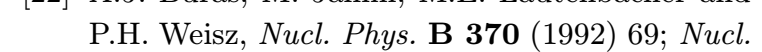
ᄂニニニン-

- - - P.

[23] A.J. Buras, M. Jamin and M.E. Lautenbacher, 'Nucl. Phys. B 400 (1993) 75 .

[24] M. Ciuchini, E. Franco, G. Martinelli and L. Reina, Phys. Lett._B_301 (1993) 263i, iNucl.' - - _ Phys. B $\mathbf{4 1 5}(1994) 403$.

[25] A.J. Buras, M. Jamin and P.H. Weisz, 'N - - _ Phys. B $\mathbf{3 4 7}(1990) 491$.

[26] S. Herrlich and U. Nierste, iNucl. Phys. B $\mathbf{4 1 9}$ (1994) 292; 'Phys. Rev. D 52 (1995) 6505 ri Nucl. Phys. B $\mathbf{4 7 6}(1996) 27$.

[27] J. Urban, F. Krauss, U. Jentschura and G. Soff, iNucl. Phys. B $\mathbf{5 2 3}(1998) 40$.

[28] A.J. Buras, hep-ph/9908395, talk given at KAON 99, (Chicago, June 1999).

[29] G. Martinelli, these proceedings.
[30] R. Gupta, T. Bhattacharaya and S.R. Sharpe, Phys. Rev. D $\mathbf{5 5}(1997)$ 4036; G. Kilcup, R. Gupta and S.R. Sharpe, Phys. Rev. D 57 (1998) 1654; L. Conti et al., 'Phys. Lett. B.421 (1998)! 273

[31] T. Hambye, G.O. Köhler, E.A. Paschos, P.H. Soldan and W.A. Bardeen, Phys. Rev. D $\mathbf{5 8}$ (1998) 014017, T. Hambye, G.O. Köhler and P.H. Soldan, hep-ph/9902334!

[32] S. Bertolini, J.O. Eeg, and M. Fabbrichesi, 'iNucl.' Phys. B 476 (1996) 749'; S. Bertolini, J.O. Eeg, M. Fabbrichesi and E.I. Lashin, iNucl. Phys. $\mathbf{B}_{1}^{\prime}$ 514 (1998) 93; S. Bertolini, J.O. Eeg, and M. Fabbrichesi, hep-ph/9802405, to appear in Rev. Mod. Phys..

[33] D. Pekurovsky and G. Kilcup, hep-lat/9812019'.

[34] M. Ciuchini, E. Franco, L. Giusti, V. Lubicz and G. Martinelli, hep-ph/9910236",

[35] T. Hambye, G.O. Köhler, E.A. Paschos and P.H. Soldan, hep-ph/9906434!.

[36] A.J. Buras, M. Jamin and M.E. Lautenbacher, 'Phys. Lett. B 389 (1996) 749,.

[37] A. Ali and D. London, Eur. Phys. J. C 9 (1999) 687; F. Parodi, P. Roudeau and A. Stocchi, hep-ex/9903063; S. Plaszcyzynski, these proceedings.

[38] J. Bijnens and J. Prades, 'J. High Energy Phys.i 01 (1999) 023; J. Prades, hep-ph/9909245; J. Bijnens, hep-ph/9910415,

[39] S. Gardner and G. Valencia, 'Phys. Lett. B $\mathbf{4 6 6}$ (1999) 355.

[40] G. Ecker, J. Gasser, A. Pich and E. de Rafael, 'Nucl. Phys. B 321 (1989) 311; G. Ecker et al., Phys. Lett. B 223 (1989) 425.

[41] E. Pallante and A. Pich, hep-ph/9911233'.

[42] R. Omnès, 'Nuovo Cim. 8 (1958)_316'.

[43] A. Masiero, these proceedings. 